\title{
LABORATORY OBSERVATIONS OF ELECTRON TEMPERATURE IN THE WAKE OF A SPHERE IN A STREAMING PLASMA
}

\author{
W. A. ORAN,* U. SAMIR, $†$ N. H. STONE* and E. G. FONTHEIM $\dagger$
}

\author{
(Received in final form 19 December 1974)
}

\begin{abstract}
A parametric study was performed of electron temperature variation in the wake of a conducting sphere in a streaming plasma. The flow conditions were varied as follows: the ambient electron temperatures in the range $850-2450 \mathrm{~K}$; the ambient electron densities in the range $5 \times 10^{4}-$ $7 \times 10^{5} / \mathrm{cm}^{3}$; and body potentials relative to plasma potential in the range of +1.7 to $-2.8 \mathrm{~V}$ for an ion beam energy of $\sim 4 \mathrm{eV}$. Electron temperature enhancements were observed which ranged up to 200 per cent above ambient in the nearest proximity of the body surface. The magnitude of the enhancement depends upon the ambient density, temperature and body potential.
\end{abstract}

\section{INTRODUCTION}

The interaction between a spacecraft and its environmental space-plasma, in particular the charged particle distribution around ionospheric satellites, has been the topic of numerous theoretical investigations in the past decade (for a recent review, see Gurevich et al., 1969). The available in situ results, on the other hand, contain a fragmentary and limited amount of information on the electron distributions at specific locations in the near wake zone. Even less information is available on the ion distributions (for a recent review, see Samir, 1973). Angular distributions of electron temperature in the closest vicinity of some spacecraft have also been presented by Berthelier and Sturges (1967), Medved (1969) and Samir and Wrenn (1972).

Recently, variations of electron temperature at the surface of a body-probe were studied by Illiano and Storey (1974) in the laboratory. Preliminary transverse profiles of electron temperature in the near wake region behind a sphere in laboratory streaming plasmas were reported by Samir et al. (1974). Here we present a parametric study of the dependence of the electron temperature in the wake upon ambient electron temperature, density, and body potential.

\section{DESCRIPTION OF THE EXPERIMENT}

Transverse and axial profiles of electron temperature, $T_{e}$, behind a stainless steel spherical target

\footnotetext{
- Space Sciences Laboratory, Marshall Space Flight Center, Huntsville, Alabama, U.S.A.

† Space Physics Research Laboratory, University of Michigan, Ann Arbor, Michigan, U.S.A.

‡Department of Environmental Sciences, Tel-Aviv University, Israel.
}

body of radius $R_{o}=4 \mathrm{~cm}$ were measured in an $\mathrm{Ar}^{+}$plasma beam at a background pressure of $4 \times 10^{-6}$ Torr. The plasma is produced with a modified Kauffman ion thruster whose small orifice effectively yields a "cold" ion beam. (The transverse ion temperature is estimated at $\sim 300 \mathrm{~K}$.) Additional details of the experimental set up can be found in Oran et al. (1974).

The measurements were made with a stainless steel, cylindrical Langmuir probe, $1.5 \mathrm{~cm}$ long and $0.1 \mathrm{~cm}$ dia., oriented perpendicular to the flow direction. The probe was bombarded with a high energy ion beam before each run to remove surface contamination. In addition, prior to and at the conclusion of each run, the $i-v$ characteristics of the probe were checked to insure that there was no "hysteresis" present.

It should be noted that the term "electron tem" perature" used herein refers to the numerical values obtained in the conventional manner by a straightline fit to the current-voltage characteristics. The decrease of $I_{e}$ in the wake region was compensated to some degree (in recording the $i-v$ curves) by increasing an amplifier gain. Hence, a straightline-fit was obtained over typically 1.5 decades both for the ambient and the wake measurements.

The authors are aware of the possibility that the electron distribution in the wake may not be truly represented by a Maxwellian. Hence, the use of the term "temperature" in the conventional manner may not be justified. However, no such deviations were detected in the slopes of the currentvoltage characteristics used in the present study which may indicate no significant departures from a Maxwellian. A more detailed discussion on the above is given in Samir and Wrenn (1972) and Illiano and Storey (1974). 


\section{RESULTS AND DISCUSSION}

We present profiles of the ratio $R\left(T_{e}\right)=T_{e w} / T_{e o}$, where $T_{e w}=$ electron temperature in the wake and $T_{e o}=$ ambient electron temperature.

Ambient conditions were determined by mapping the flow field with the target body removed from the flow. All distances are normalized to the sphere radius, and the values of the flow conditions are shown in the figures. Here $N_{o}$ is the ambient plasma density, $E_{i}$ the ion beam energy, $\phi_{s}$ the body potential relative to plasma potential, and $\lambda_{D}=$ $6.9\left(T_{e o} / N_{o}\right)^{1 / 2} \mathrm{~cm}$ is the Debye length. The experimental error for each value of $R\left(T_{e}\right)$ is $\leqslant 20$ per cent.

Figure 1 presents examples of axial profiles of $R\left(T_{e}\right)$ at different locations downstream from the centre of the sphere $\left(r / R_{0}\right)$ for body potentials $\phi_{s}$ in the range $+0.8--1.2 \mathrm{~V}$. It can be seen that an electron temperature enhancement significantly above the experimental error exists in the very near wake.

Examining the general influence of $\phi_{s}$ on $R\left(T_{e}\right)$, we see that at a distance of $1.25 R_{o}$ from the centre of the sphere (on the wake axis) the enhancements can be as great as 3 times $T_{e o}$ for the more negative body potentials (see Fig. 1). For these potentials, however, the $R\left(T_{e}\right)$ enhancement seems to be confined to distances $\left(r / R_{0}\right) \leqslant 2$. For more positive values of $\phi_{s}$, the enhancement appears to extend further downstream, although the maximum value of the enhancement is much smaller.

Figure 2 presents examples of transverse profiles

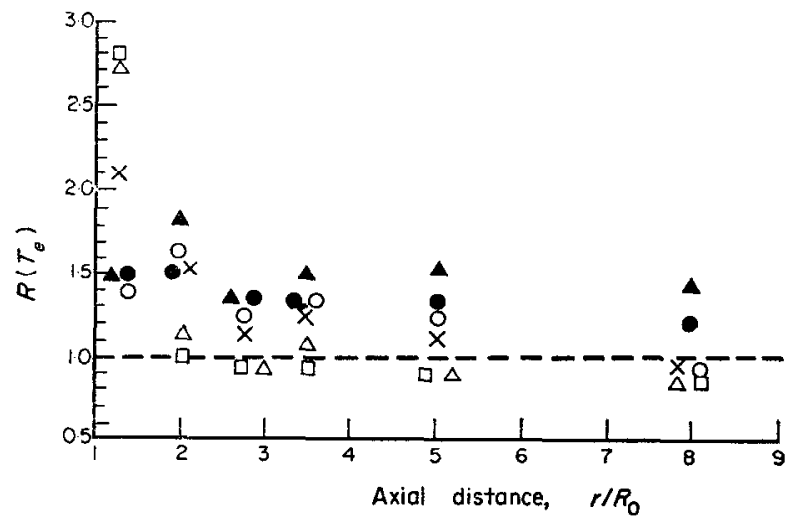

Fig. 1. AxIal PRofiles of $R\left(T_{e}\right)=T_{e v} / T_{e o}$ AT DIFFerent DOWNSTREAM DISTANCES $\left(r / R_{0}\right)$ BEHIND THE SPHERE AND FOR DIFFERENT BODY POTENTIALS, $\phi_{s}$ : $A \sim+0.8 \mathrm{~V}$, $0 \sim+0.3 \mathrm{~V}, \quad 0 \sim 0 \mathrm{~V}, \mathrm{x} \sim-0.3 \mathrm{~V}, \Delta \sim-0.7 \mathrm{~V}$, AND $\square \sim-1 \cdot 2 \mathrm{~V}$.

The ambient plasma conditions are: electron temperature $\left(T_{e 0}\right) \sim 1050 \mathrm{~K}$, density $\left(N_{0}\right) \sim 6 \times 10^{4} / \mathrm{cm}^{3}$, ion beam energy $\left(E_{i}\right) \sim 3.2 \mathrm{eV}$ and Debye length $\left(\lambda_{D}\right) \sim$ $0.9 \mathrm{~cm}$.
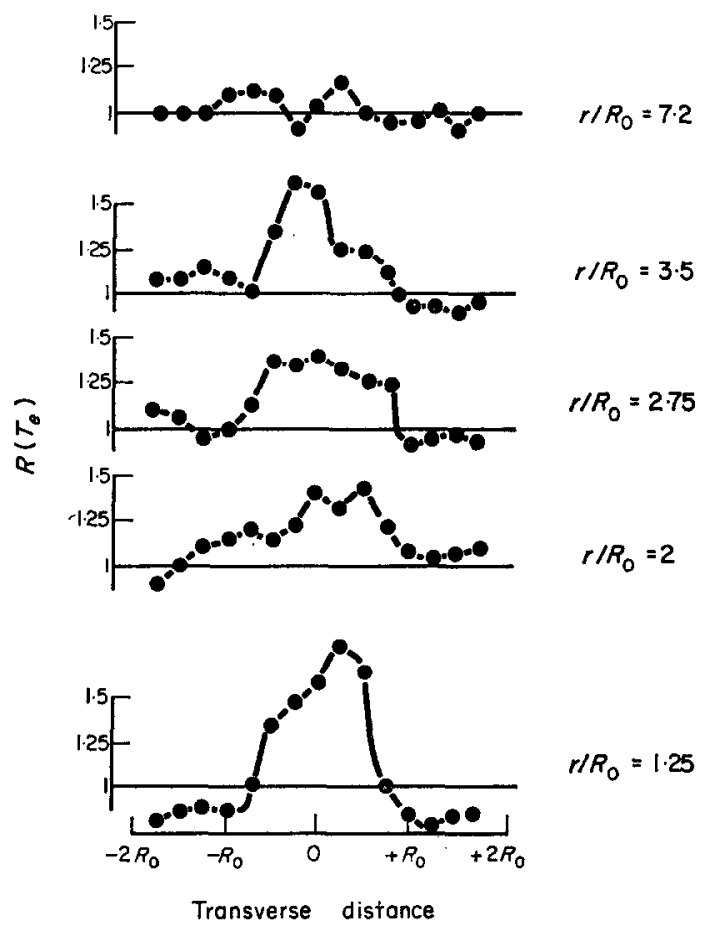

Fig. 2. Transverse propiles of $R\left(T_{e}\right)=T_{e \omega} / T_{e 0}$ AT DIFFERENT DOWNSTREAM UISIANCES $\left(r / R_{\bullet}\right)$ BEHIND THE SPHERE WHICH WAS AT FLOATING POTENTIAL $\left(\phi_{S} \sim\right.$ $-0.3 \mathrm{~V})$.

The ambient plasma conditions are: electron temperature $\left(T_{e 0}\right) \sim 1200 \mathrm{~K}$, density $\left(N_{o}\right) \sim 7.5 \times 10^{4} / \mathrm{cm}^{3}$, ion beam energy $\left(E_{i}\right) \sim 5.3 \mathrm{eV}$ and Debye length $\left(\lambda_{D}\right) \sim$ $0.9 \mathrm{~cm}$. After Samir et al. (1974).

of $R\left(T_{e}\right)$ at different downstream locations. The plasma flow conditions are similar to those in Fig. 1, although in this instance the body was at floating potential $(\sim-0.3 \mathrm{~V})$. Significant electron temperature enhancemnts are also observed in the near wake. The transverse extent of the enhancement appears to be on the order of the sphere diameter.

Figure 3 presents axial profiles of $R\left(T_{e}\right)$. In this case, the ambient density $N_{o}$ is larger by about an order of magitude compared with the density given in Fig. 1. The rest of the flow conditions are similar. Here no enhancement in $R\left(T_{e}\right)$ can be observed to within the experimental error. Hence the dependence of $T_{e w}$ on $N_{o}$ is clearly demonstrated. Figure 3 also indicates that at the ambient density $7 \times 10^{5} / \mathrm{cm}^{3}$, the $T_{e}$ enhancement is relatively insensitive to the variations of $\phi_{s}$ over the range of $-1.2-+0.8 \mathrm{~V}$. This is in contrast to the strong $\phi_{s}$ dependence of $R\left(T_{e}\right)$ for $N_{o}=6 \times 10^{4} / \mathrm{cm}^{3}$ (see Fig. 1), in the near wake.

Figure 4 presents axial profiles of $R\left(T_{e}\right)$ where 


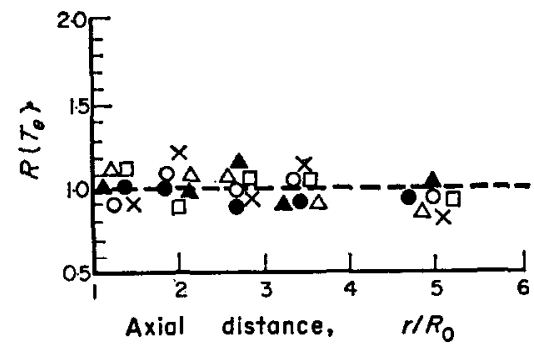

Fig. 3. AXIAL PROFILES OF $R\left(T_{e}\right)=T_{e w} / T_{e o}$ AT DIFFERENT DOWNSTREAM DISTANCES $\left(r / R_{0}\right)$ BEHIND THE SPHERE FOR DIFFERENT BODY POTENTIALS, $\phi_{s}: \Delta \sim+0.8 \mathrm{~V}, \bullet \sim$ $+0.3 \mathrm{~V}, \quad 0 \sim 0 \mathrm{~V}, \mathrm{x} \sim-0.3 \mathrm{~V}, \Delta \sim-0.7 \mathrm{~V}$ AND $\square \sim-1 \cdot 2 \mathrm{~V}$.

The ambient plasma conditions are: electron temperature $\left(T_{e 0}\right) \sim 850 \mathrm{~K}$, density $\left(N_{0}\right) \sim 7 \times 10^{5} / \mathrm{cm}^{\mathrm{3}}$, ion beam energy $\left(E_{i}\right) \sim 3.6 \mathrm{eV}$ and Debye length $\left(\lambda_{D}\right) \sim$ $0.25 \mathrm{~cm}$.

$T_{e o}$ has been increased by more than a factor of 2 compared with that of Fig. 1, while the ambient density is basically the same. It is clearly seen that the effect of increasing the ambient temperature by a factor of $\sim 2$ is equivalent to increasing the ambient density by a factor of $\sim 10$.

In addition, it is interesting to note that in the case of Fig. 3, the value of $\lambda_{D}$ is decreased compared to that of Fig. 1, while the case of Fig. 4 represents

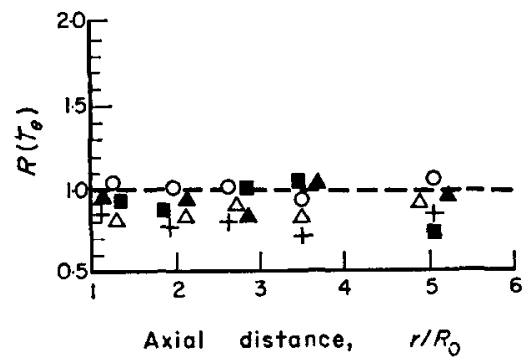

FIG. 4. Axial PROFILES of $R\left(T_{e}\right)=T_{e v} / T_{e 0}$ AT DIFFERENT DOWNSTREAM DISTANCES $\left(r / R_{0}\right)$ BEHIND THE SPHERE AND FOR DIFFERENT RODY POTENTIALS, $\phi_{\varepsilon}: \sim+1.7 \mathrm{~V}$, $\Delta \sim+0.8 \mathrm{~V}, 0 \sim 0 \mathrm{~V}, \Delta \sim-0.7 \mathrm{~V}$ AND $+\sim-2.8 \mathrm{~V}$. The ambient plasma conditions are: electron temperature $\left(T_{e 0}\right) \sim 2450 \mathrm{~K}$, density $\left(N_{0}\right) \sim 5 \times 10^{4} / \mathrm{cm}^{3}$, ion beam energy $\left(E_{i}\right) \sim 4.7 \mathrm{eV}$ and Debye length $\left(\lambda_{D}\right) \sim$ $1.55 \mathrm{~cm}$. an increase of $\lambda_{D}$. This seems to indicate that the variation of the temperature enhancement can better be described in terms of its dependence on the status of the plasma (e.g. $T_{e o}, N_{o}$ ) rather than its dependence on the parameter $\lambda_{D}$.

Our main finding, i.e. $R\left(T_{e}\right)>1$ in the very near wake at negative body potentials, is in agreement with in situ results. Moreover, the Ariel I and Explorer 31 results (Henderson and Samir, 1967; Samir and Wrenn, 1972) pointed towards the possibility that the enhancements may be confined to the very near wake. This is also seen in our results (Fig. 1 and 2).

Acknowledgements-U. Samir and E. G. Fontheim would like to acknowledge the support under grants NGR 23-005-320 and NGR 23-005-015.

\section{REFERENCES}

Berthelier, J. J. and Sturges, D. J. (1967). Simultaneous measurements of electron density and temperature in the northern auroral zone. Planet. Space Sci. 15, 1049. Gurevich, A. V., Pitaevskii, L. P. and Smirnova, V. V. (1970). Ionospheric acrodynamics. Sov. Phys. Uspekhi 12, 595. Also (1969). Space Sci. Rev. 9, 805.

Henderson, C. L. and Samir, U. (1967). Observations of the disturbed region around an ionospheric spacecraft. Planet. Space Sci. 15, 1499.

Illiano, J. M. and Storey, L. R. O. (1974). Apparent enhancement of electron temperature in the wake of a spherical probe in a flowing plasma. Planet. Space Sci. 22, 873 .

Medved, D. B. (1969). Measurement of ion wakes and body effects with the Gemini/Agena satellitc. Rarefied Gas Dynamics, 6th Int. Symp., p. 1525.

Oran, W. A., Samir, U. and Stone, N. H. (1974). Parametric study of the near-wake structure of spherical and cylindrical bodies in the laboratory. Planet. Space Sci. 22, 379.

Samir, U. (1973). Charged particle distribution in the nearest vicinity of ionospheric satellites, comparison of the main results from the Ariel I, Explorer 31, and Gemini/Agena 10 spacecraft. Particle and Photon Interactions with Surfaces in Space (Ed. G. Grard), p. 154. North Holland, Amsterdam.

Samir, U. and Wrenn, G. L. (1972). Experimental evidence of an electron temperature enhancement in the wake of an ionospheric satellite. Planet. Space Sci. $20,899$.

Samir, U., Oran, W. A. and Stone, N. H. (1974). Laboratory simulation of space aerodynamic phenomena: satellite wake studies. Rarefied Gas Dynamics, 9th Symp. July 1974 (D.11). 